\title{
Risk factors of clinical birth asphyxia and subsequent newborn death following nuchal cord in a low-resource setting ${ }^{*}$
}

\author{
Pascal Foumane ${ }^{1,2 \#}$, Gustave Nkomom ${ }^{1}$, Emile Telesphore Mboudou ${ }^{1,2}$, Julius Dohbit Sama ${ }^{1,2}$, \\ Séraphin Nguefack ${ }^{3}$, Boniface Moifo ${ }^{4}$
}

${ }^{1}$ Department of Obstetrics and Gynecology, Faculty of Medicine and Biomedical Sciences (FMBS), The University of Yaoundé 1, Yaoundé, Cameroon

${ }^{2}$ Department of Obstetrics and Gynecology, Yaoundé Gyneco-Obstetric and Pediatric Hospital, Yaoundé, Cameroon

${ }^{3}$ Department of Pediatrics, Yaoundé Gyneco-Obstetric and Pediatric Hospital, Yaoundé, Cameroon

${ }^{4}$ Department of Radiology, Yaoundé Gyneco-Obstetric and Pediatric Hospital, Yaoundé, Cameroon

Email: "pfoumane2004@yahoo.fr

Received 24 September 2013; revised 20 October 2013; accepted 28 October 2013

Copyright (C) 2013 Pascal Foumane et al. This is an open access article distributed under the Creative Commons Attribution License, which permits unrestricted use, distribution, and reproduction in any medium, provided the original work is properly cited.

\begin{abstract}
Introduction: Our aim was to identify the risk factors of clinical birth asphyxia and subsequent newborn death in the presence of nuchal cord in a sub-Saharan Africa setting. Methodology: It was a six-months' case-control study involving 117 parturients whose babies presented with a nuchal cord at delivery. The study was carried out at the Yaoundé Gyneco-Obstetric and Pediatric Hospital, Cameroon, from January $1^{\text {st }}$ to June $30^{\text {th }}$ 2013. Results: The risk factors of clinical birth asphyxia identified were: first delivery, absence of obstetrical ultrasound during pregnancy, nuchal cord with more than one loop, duration of second stage of labor more than $\mathbf{3 0}$ minutes during vaginal delivery. The risk factors for newborn death from clinical birth asphyxia in the presence of nuchal cord were: maternal age $<20$ years, first delivery, absence of obstetrical ultrasound during pregnancy, nuchal cord with more than one loop, tight nuchal cord, duration of second stage of labor more than 30 minutes during vaginal delivery. Conclusion: We recommend a systematic obstetrical ultrasound before labor, so as to detect the presence of a nuchal cord, its tightness and the number of loops. Also, cesarean section should be considered when a nuchal cord is associated with first delivery, tightness or multiple looping.
\end{abstract}

Keywords: Risk Factors; Nuchal Cord; Adverse Outcome; Birth Asphyxia; Newborn Death; Apgar

*Declaration of interest: The authors report no declaration of interest.

${ }^{\#}$ Corresponding author.
Score; Cameroon

\section{INTRODUCTION}

Nuchal cord is of common occurrence at delivery. The reported incidence varies from $5.7 \%$ in India to up to $35.1 \%$ in Switzerland [1,2]. In Cameroon, the published incidence of nuchal cord at delivery also varies from $5.5 \%$ to $16.2 \%$ according to authors $[3,4]$. Even though many authors have concluded that nuchal cord is not associated with adverse outcome [5-7], most of the studies reported birth asphyxia and perinatal death related to nuchal cord [1,2,8-10]. SHAFFER et al. in Switzerland [2], and LARSON et al. in USA [8], have experienced unfavorable neonatal blood gas values at birth when nuchal cord was present. Fetal distress with low fifth minute Apgar scores and perinatal deaths in cases of nuchal cord has been reported worldwide $[1,9,10]$. At the same time, apart from tight nuchal cord that has been shown to be associated with fetal distress, operative delivery and perinatal death [1], little is known concerning the risk factors of birth adverse outcome in the presence of nuchal cord, especially in a sub-Saharan Africa setting. The identification of these factors in our setting is an important step in reducing perinatal morbidity and mortality related to nuchal cord. The objective of this study was therefore to identify the risk factors of clinical neonatal asphyxia and subsequent newborn death in the presence of nuchal cord.

\section{METHODS}

This case-control study involved 117 parturients whose 
babies presented with a nuchal cord at delivery at the Yaoundé Gyneco-Obstetric and Pediatric Hospital, Cameroon. After the approval of the protocol by the ethical committee of the hospital, all the parturients with babies presenting with a nuchal cord at delivery, from January $1^{\text {st }}$ to June $30^{\text {th }} 2013$, were consecutively recruited. Intrauterine fetal death before labor was an exclusion criteria. Each woman was counseled about the study and her consent was obtained prior to recruitment. A pretested questionnaire was administered by an investigator and information was retrieved from the patients' files. The variables of interest were: maternal age, parity, gestational age, ultrasound during pregnancy, mode of delivery, duration of expulsion in case of vaginal delivery, number of nuchal cord loops, type of nuchal cord (tight or loose), fetal weight, fetal sex, fifth minute Apgar score and newborn death following clinical asphyxia. Clinical birth asphyxia was considered when the fifth minute Apgar score was $<7$. To identify the risk factors of clinical neonatal asphyxia, the parturients whose babies had clinical asphyxia at birth (case group) were compared to those with babies having a fifth minute Apgar score $\geq 7$ (control group). The risk factors of newborn death following clinical asphyxia were identified by comparing parturients with newborn death following clinical asphyxia at birth (case group) to those without newborn death (control group). The calculated minimal sample size was 6 subjects for each group, based on the $0.2 \%$ of newborn death due to nuchal cord among 3136 deliveries reported by DHAR et al. [1]. The chosen precision for statistical calculations was 5\%. Statistical analysis was done using Epi info 3.5.3 software. The difference was statistically significant for $P<0.05$. Pearson's Chi square and Fisher's exact test were used to compare proportions. Odds ratio (OR) and its 95\% Confidence Interval (CI) were calculated to assess the association between the variables and clinical birth asphyxia as well as subsequent newborn death.

\section{RESULTS}

During the six months' recruitment period, we recorded 1405 deliveries of which 117 fetuses presented a nuchal cord at birth, giving an incidence of nuchal cord at birth of $8.3 \%$. Only one twin pregnancy was encountered with nuchal cord for the second twin. Of the 117 cases of nuchal cord, 16 neonates $(16 / 117 ; 13.7 \%)$ had a fifth minute Apgar score $<7$. The incidence of clinical birth asphyxia in nuchal cord cases was $13.7 \%$. Among the 16 neonates with poor fifth minute Apgar score, 8 (8/117; $6.8 \%$ ) died in spite of resuscitation efforts, giving an incidence of newborn death following birth of $6.8 \%$ among neonates born with a nuchal cord and presenting clinical neonatal asphyxia.

The risk factors of clinical birth asphyxia identified using bivariate analysis were (Table 1): first delivery $(P$ $=0.007 ; \mathrm{OR}=4.1 ; \mathrm{CI}=1.4-12.4)$, absence of obstetrical ultrasound during pregnancy $(P=0.02 ; \mathrm{OR}=4.5 ; \mathrm{CI}=$ $1.2-16.0)$, nuchal cord with more than one loop $(P=$ $0.02 ; \mathrm{OR}=3.5 ; \mathrm{CI}=1.2-10.4)$, duration of second stage of labor more than 30 minutes during vaginal delivery $(P$ $<0.001 ; \mathrm{OR}=22.2 ; \mathrm{CI}=6.7-73$ ).

Protective factors of clinical birth asphyxia in nuchal cord were (Table 1): parity $\geq 2(P=0.007 ; \mathrm{OR}=0.2$; CI $=0.08-0.7)$, obstetrical ultrasound during pregnancy $(P$ $=0.02 ; \mathrm{OR}=0.2 ; \mathrm{CI}=0.06-0.8)$, nuchal cord with only one loop $(P=0.02 ; \mathrm{OR}=0.3 ; \mathrm{CI}=0.09-0.8)$, duration of expulsion of 30 minutes or less during vaginal delivery $(P<0.001 ; \mathrm{OR}=0.05 ; \mathrm{CI}=0.01-0.2)$.

The risk factors for newborn death from clinical birth asphyxia in case of nuchal cord were (Table 2): maternal age $<20$ years $(P<0.001 ; \mathrm{OR}=12.5 ; \mathrm{CI}=3.1-50.1)$, first delivery $(P=0.002 ; \mathrm{OR}=16.8 ; \mathrm{CI}=3.3-87.1)$, absence of obstetrical ultrasound during pregnancy $(P=$ $0.03 ; \mathrm{OR}=7.6 ; \mathrm{CI}=1.9-30.0)$, nuchal cord with more than one loop $(P=0.01 ; \mathrm{OR}=7.9 ; \mathrm{CI}=1.9-33)$, tight nuchal cord $(P=0.009 ; \mathrm{OR}=8.3 ; \mathrm{CI}=2.0-34.8)$, duration of second stage of labor more than 30 minutes during vaginal delivery $(P=0.002 ; \mathrm{OR}=22.9 ; \mathrm{CI}=5.5$ 95.1).

Protective factors of newborn death following clinical neonatal asphyxia in nuchal cord were (Table 2): second delivery or more $(P=0.002 ; \mathrm{OR}=0.06 ; \mathrm{CI}=0.01-0.3)$, obstetrical ultrasound during pregnancy $(P=0.03 ; \mathrm{OR}=$ $0.13 ; \mathrm{CI}=0.03-0.5)$, nuchal cord with only one loop ( $P$ $=0.01 ; \mathrm{OR}=0.13 ; \mathrm{CI}=0.02-0.6)$, loose nuchal cord $(P$ $=0.009 ; \mathrm{OR}=0.12 ; \mathrm{CI}=0,02-0,6)$, duration of expulsion of 30 minutes or less during vaginal delivery $(P<$ $0.002 ; \mathrm{OR}=0.04 ; \mathrm{CI}=0.01-0.2$ ).

Among the 93 mothers who delivered vaginally, first delivery was significantly associated with second stage of labor lasting more than 30 minutes $(P<0.001 ; \mathrm{OR}=$ 19.2; $\mathrm{CI}=5.7-64.7)$.

\section{DISCUSSION}

Maternal age less than 20 years is identified as a risk factor of newborn death from clinical birth asphyxia. This factor is not commonly found in the available literature. In fact, it seems more a confounding factor than an independent risk factor. When a delivery takes place at maternal age less than 20 years, it is usually the first experience in a woman's reproductive life. Therefore, primiparity appears to be the factor explaining this finding.

According to our findings, the first delivery exposes to clinical birth asphyxia and subsequent newborn death when a nuchal cord is observed. RHOADES (10) et al. have reported an association between an increased risk of nuchal cord and primiparity in USA, while GHI et al. 
Table 1. Comparison of the variables between the group with clinical birth asphyxia (case group; $n=16$ ) and the group without clinical birth asphyxia (control group; $n=101$ ).

\begin{tabular}{|c|c|c|c|c|}
\hline Variables & Clinical birth asphyxia $n(\%)$ & No clinical birth asphyxia $n$ (\%) & $\boldsymbol{P}$ & Odds ratio $\left(95 \% \mathrm{CI}^{*}\right)$ \\
\hline \multicolumn{5}{|l|}{ Maternal age } \\
\hline$<20$ & $3(18.7)$ & $5(4.9)$ & 0.07 & $4.4(0.9-20.7)$ \\
\hline $20-30$ & $8((50.0)$ & $53(52.5)$ & 0.90 & $0.9(0.3-2.6)$ \\
\hline $30-40$ & $5(31.3)$ & $43(42.6)$ & 0.40 & $0.6(0.2-1.9)$ \\
\hline \multicolumn{5}{|l|}{ Parity } \\
\hline 1 & $10(62.5)$ & $29(28.7)$ & \multirow{2}{*}{0.007} & $4.1(1.4-12.4)$ \\
\hline$\geq 2$ & $6(37.5)$ & $72(71.3)$ & & $0.2(0.08-0.7)$ \\
\hline \multicolumn{5}{|l|}{ Gestational age } \\
\hline $28-37$ & $2(12.5)$ & $5(4.9)$ & 0.20 & $2.7(0.5-15.5)$ \\
\hline $37-40$ & $9(56.2)$ & $54(53.5)$ & 0.80 & $1.1(0.4-3.2)$ \\
\hline$>40$ & $5(31.3)$ & $42(41.6)$ & 0.40 & $0.6(0.2-2.0)$ \\
\hline \multicolumn{5}{|c|}{ Obstetrical ultrasound during pregnancy } \\
\hline none & $4(25.0)$ & $7(6.9)$ & \multirow{2}{*}{0.02} & $4.5(1.2-16.0)$ \\
\hline One or more & $12(75.0)$ & $94(93.1)$ & & $0.2(0.06-0.8)$ \\
\hline \multicolumn{5}{|l|}{ Mode of delivery } \\
\hline Vaginal & $13(81.2)$ & $80(79.2)$ & \multirow{2}{*}{0.80} & $1.14(0.3-4.4)$ \\
\hline Cesarean & $3(18.8)$ & $21(20.8)$ & & $0.9(0.2-3.4)$ \\
\hline \multicolumn{5}{|l|}{ Number of loops } \\
\hline 1 & $7(43.7)$ & $74(73.3)$ & \multirow{2}{*}{0.02} & $0.3(0.09-0.8)$ \\
\hline$\geq 2$ & $9(56.3)$ & $27(26.7)$ & & $3.5(1.2-10.4)$ \\
\hline \multicolumn{5}{|l|}{ Type of nuchal cord } \\
\hline Tight & $9(56.3)$ & $74(73.3)$ & \multirow{2}{*}{0.16} & $2.1(0.7-6.3)$ \\
\hline Loose & $7(43.7)$ & $27(26.7)$ & & $0.5(0.16-1.4)$ \\
\hline \multicolumn{5}{|l|}{ Fetal weight } \\
\hline$<2500 \mathrm{~g}$ & $2(12.5)$ & $12(11.9)$ & 0.92 & $1.1(0.2-5.2)$ \\
\hline $2500 \mathrm{~g}-3500 \mathrm{~g}$ & $9(56.2)$ & $56(55.4)$ & 0.53 & $1.4(0.4-4.6)$ \\
\hline$>3500 \mathrm{~g}$ & $5(31.3)$ & $33(32.7)$ & 0.92 & $0.9(0,3-2,9)$ \\
\hline \multicolumn{5}{|l|}{ Fetal sex } \\
\hline Female & $9(56.3)$ & $46(45.5)$ & \multirow{2}{*}{0.42} & $1.5(0.5-4.4)$ \\
\hline Male & $7(43.7)$ & $55(54.5)$ & & $0.6(0.2-1.9)$ \\
\hline \multicolumn{5}{|c|}{ Length of umbilical cord (cm) } \\
\hline 30 to 70 & $10(62.5)$ & $78(77.2)$ & \multirow{2}{*}{0.20} & $0.5(0.16-1.5)$ \\
\hline$\geq 70$ & $6(37.5)$ & $23(22.8)$ & & $2.0(0.7-6.2)$ \\
\hline \multicolumn{5}{|c|}{ Duration of $2^{\text {nd }}$ stage of labor for vaginal deliveries } \\
\hline$>30$ minutes & $7(53.8)$ & $4(5.0)$ & \multirow{2}{*}{0.001} & $22.2(6.7-73)$ \\
\hline$\leq 30$ minutes & $6(46.2)$ & $76(95.0)$ & & $0.05(0.01-0.2)$ \\
\hline
\end{tabular}

${ }^{*} \mathrm{CI}=$ Confidence Interval. 
Table 2. Comparison of the variables between the group with newborn death following clinical birth asphyxia (case group; $n=8$ ) and the group without newborn death (control group; $n=109$ ).

\begin{tabular}{|c|c|c|c|c|}
\hline Variables & newborn death $n(\%)$ & No newborn death $n(\%)$ & $\mathbf{P}$ & Odds ratio $\left(95 \% \mathrm{CI}^{*}\right)$ \\
\hline \multicolumn{5}{|l|}{ Maternal age } \\
\hline$<20$ & $3(37.5)$ & $5(4.6)$ & 0.001 & $12.5(3.1-50.1)$ \\
\hline $20-30$ & $3((37.5)$ & $58(53.2)$ & 0.31 & $0.5(0.12-2.3)$ \\
\hline $30-40$ & $2(25.0)$ & $46(42.2)$ & 0.29 & $0.46(0.1-2.3)$ \\
\hline \multicolumn{5}{|l|}{ Parity } \\
\hline $1\left(1^{\text {st }}\right.$ delivery $)$ & $7(87.5)$ & $32(29.4)$ & \multirow[b]{2}{*}{0.002} & $16.8(3.3-87.1)$ \\
\hline$\geq 2\left(2^{\text {nd }}\right.$ delivery or more $)$ & $1(12.5)$ & $77(70.6)$ & & $0.06(0.01-0.3)$ \\
\hline \multicolumn{5}{|l|}{ Gestational age } \\
\hline $28-37$ & $2(25.0)$ & $5(4.6)$ & 0.07 & $6.9(0.9-27.1)$ \\
\hline $37-40$ & $4(50.0)$ & $59(54.1)$ & 0.72 & $0.8(0.2-3.5)$ \\
\hline$>40$ & $2(25.0)$ & $45(41.3)$ & 0.30 & $0.47(0.1-2.4)$ \\
\hline \multicolumn{5}{|c|}{ Obstetrical ultrasound during pregnancy } \\
\hline none & $3(37.5)$ & $8(7.4)$ & \multirow{2}{*}{0.03} & $7.6(1.9-30.0)$ \\
\hline One or more & $5(62.5)$ & $101(92.6)$ & & $0.13(0.03-0.5)$ \\
\hline \multicolumn{5}{|l|}{ Mode of delivery } \\
\hline Vaginal & $6(75.0)$ & $87(79.8)$ & \multirow{2}{*}{0.52} & $0.7(0.1-4.3)$ \\
\hline Cesarean & $2(25.0)$ & $22(10.2)$ & & $1.3(0.2-6.9)$ \\
\hline \multicolumn{5}{|l|}{ Number of loops } \\
\hline 1 & $2(43.7)$ & $79(73.3)$ & \multirow{2}{*}{0.01} & $0.13(0.02-0.6)$ \\
\hline$\geq 2$ & $6(56.3)$ & $30(26.7)$ & & $7.9(1.9-33)$ \\
\hline \multicolumn{5}{|l|}{ Type of nuchal cord } \\
\hline Tight & $6(75.0)$ & $29(26.6)$ & \multirow{2}{*}{0.009} & $8.3(2.0-34.8)$ \\
\hline Loose & $2(25.0)$ & $80(73.4)$ & & $0.12(0.02-0.6)$ \\
\hline \multicolumn{5}{|l|}{ Fetal weight } \\
\hline$<2500 \mathrm{~g}$ & $1(12.5)$ & $13(11.9)$ & 0.75 & $1.05(0.1-9.2)$ \\
\hline $2500 \mathrm{~g}-3500 \mathrm{~g}$ & $5(62.5)$ & $60(55.0)$ & 0.49 & $1.4(0.3-5.9)$ \\
\hline$>3500 \mathrm{~g}$ & $2(25.0)$ & $36(33.1)$ & 0.48 & $0.7(0.1-3.5)$ \\
\hline \multicolumn{5}{|l|}{ Fetal sex } \\
\hline Female & $3(37.5)$ & $52(47.7)$ & \multirow{2}{*}{0.31} & $0.66(0.15-2.8)$ \\
\hline Male & $5(62.5)$ & $57(52.3)$ & & $1.5(0.3-6.6)$ \\
\hline \multicolumn{5}{|l|}{ Length of umbilical cord (cm) } \\
\hline 30 to 70 & $5(62.5)$ & $83(76.1)$ & \multirow{2}{*}{0.31} & $0.5(0.1-2.3)$ \\
\hline$\geq 70$ & $3(37.5)$ & $26(13.9)$ & & $1.9(0.4-8.5)$ \\
\hline \multicolumn{5}{|c|}{ Duration of $2^{\text {nd }}$ stage of labor for vaginal deliveries } \\
\hline$>30$ minutes & $4(66.7)$ & $7(8.0)$ & \multirow{2}{*}{0.002} & $22.9(5.5-95.1)$ \\
\hline$\leq 30$ minutes & $2(33.3)$ & $80(92.0)$ & & $0.04(0.01-0.2)$ \\
\hline
\end{tabular}

${ }^{*} \mathrm{CI}=$ Confidence Interval. 
[11] have found that parity $\geq 1$ before delivery was associated with success of induction in Italy. We also found that primiparity was significantly associated to a second stage of labor lasting more than 30 minutes, which itself was a significant exposure to clinical birth asphyxia and subsequent newborn death. These findings are similar to those of OGUEH et al. [12] who found a significant association between primiparous with tight nuchal cord and prolonged second stage of labor in Canada. Therefore, prolonged second stage of labor can explain the role of primiparity as a risk factor of birth adverse outcome in this study.

The absence of obstetrical ultrasound is a risk factor of both clinical birth asphyxia and subsequent newborn death. This is a finding that is probably specific to our milieu. In developed countries, all pregnant women have at least one obstetrical ultrasound done before delivery [13]. This is not the case in our study. Even though GONZÁLEZ-QUINTERO et al. noted that a sonographically detected nuchal cord is not associated with important perinatal complications [14], the practice of obstetrical ultrasound gives the awareness on the presence of nuchal cord with some characteristics such as the type (tight or loose) and the number of loops, which are identified in this study as risk factors for adverse outcome. On the other hand, the increased risk of cesarean section in nuchal cord cases reported by some authors in Western Countries [15] is probably and at least partially explained by the routine third trimester obstetrical ultrasound.

Nuchal cord with more than one loop is found a risk factor of clinical birth asphyxia and subsequent newborn death. This is similar to the findings of JAUNIAUX et al. in Belgium, who reported that multiple looping of the umbilical cord around the fetal neck was the main factor associated with meconium-stained amniotic fluid, emergency cesarean section, need for neonatal resuscitation and admission to the neonatal intensive care unit when nuchal cord was documented at delivery [16]. LARSON et al. also found in Oklahoma City, USA, that a multiple looping of nuchal cord was associated with abnormal fetal heart rate pattern during advanced labor, low or midforceps application, meconium-stained amniotic fluid and an abnormal umbilical artery $\mathrm{pH}$ [8]. In fact, multiple loops shorten the cord length and probably exposes to cord traction and compression.

In this study, tight nuchal cord was a risk factor of newborn death from clinical birth asphyxia. Tight nuchal cord is the commonest factor associated with adverse neonatal outcome. When comparing tight nuchal cord to loose nuchal cord in an Indian hospital, DHAR et al. found that babies with tight nuchal cord were more frequently met with fetal distress, prolonged second stage and operative delivery [1]. A Canadian study also experienced more shoulder dystocia cases when the nuchal cord was tight [12]. In Cameroon, KEMFANG NGOWA et al reported higher rates of cesarean sections and transfer of the baby to the neonatology unit in tight nuchal cord cases [4]. The same team found lower rates of emergency cesarean section and transferred to the neonatology unit amongst loose nuchal cords.

The duration of second stage of labor more than 30 minutes is found to be a risk factor to both clinical birth asphyxia and subsequent newborn death. A longer second stage of labor has been reported in nuchal cord cases, especially in parturients delivering for the first time [12] and multiple looping nuchal cords [8]. This result may help to explain the finding of primiparity as risk factor of adverse outcome in nuchal cord by the present study.

However, our results must be considered with some limitations. The number of the subjects included in the case groups is limited, just above the calculated minimal sample size for each group. Also, logistic regression was not used during analysis. All these might have given some bias to our results.

\section{CONCLUSIONS}

Maternal age $<20$ years, first delivery, absence of obstetrical ultrasound during pregnancy, nuchal cord with more than one loop, tight nuchal cord, duration of second stage of labor more than 30 minutes during vaginal delivery, are risk factors of clinical birth asphyxia and/or subsequent newborn death in nuchal cord identified in our setting.

We recommend a systematic obstetrical ultrasound before labor, so as to detect the presence of a nuchal cord, its tightness and the number of loops. Also, cesarean section should be considered when a nuchal cord is associated with first delivery, tightness or multiple looping.

These risk factors may also be used in the future to build a score that may be helpful in decision making when facing a singleton term pregnancy with a nuchal cord before delivery.

\section{ACKNOWLEDGEMENTS}

We acknowledge the authorities of Yaoundé Gyneco-Obstetric and Pediatric Hospital who gave us the permission to carry out the study in their institution.

\section{REFERENCES}

[1] Dhar, K.K., Ray, S.N. and Dhall, G.I. (1995) Significance of nuchal cord. Journal of the Indian Medical Association, 93, 451-453.

[2] Schäffer, L., Burkhardt, T., Zimmermann, R. and Kurmanavicius, J. (2005) Nuchal cords in term and postterm deliveries-Do we need to know? Obstetrics \& Gynecology, 106, 23-28. 
http://dx.doi.org/10.1097/01.AOG.0000165322.42051.0f

[3] Nkwabong, E. and Fomulu, J.N. (2011) Neonatal outcome in cases of nuchal cord in Cameroon. International Journal of Gynecology \& Obstetrics, 114, 287-288. http://dx.doi.org/10.1016/j.ijgo.2011.03.022

[4] Kemfang Ngowa, J.D., Kasia, J.M., Nsangou, I., Zedjom, C., Domkam, I., Morfaw, F., et al. (2011) Nuchal cord and perinatal outcome at the Yaounde General Hospital, Cameoon. Clinics in Mother and Child Health, 8, C101201. http://dx.doi.org/10.4303/cmch/C101201

[5] Sheiner, E., Abramowicz, J.S., Levy, A., Silberstein, T., Mazor, M. and Hershkovitz, R. (2006) Nuchal cord is not associated with adverse perinatal outcome. Archives of Gynecology and Obstetrics, 274, 81-83. http://dx.doi.org/10.1007/s00404-005-0110-2

[6] Shrestha, N.S. and Singh, N. (2007) Nuchal cord and perinatal outcome. Kathmandu University Medical Journal (KUMJ), 5, 360-363.

[7] Ghosh, G.S. and Gudmundsson, S. (2008) Nuchal cord in post-term pregnancy-relationship to suspected intrapartum fetal distress indicating operative intervention. Journal of Perinatal Medicine, 36, 142-144. http://dx.doi.org/10.1515/JPM.2008.012

[8] Larson, J.D., Rayburn, W.F., Crosby, S. and Thurnau, G.R. (1995) Multiple nuchal cord entanglements and intrapartum complications. American Journal of Obstetrics \& Gynecology, 173, 1228-1231. http://dx.doi.org/10.1016/0002-9378(95)91359-9

[9] Assimakopoulos, E., Zafrakas, M., Garmiris, P., Goulis, D.G., Athanasiadis, A.P., Dragoumis, K. and Bontis, J. (2005) Nuchal cord detected by ultrasound at term is associated with mode of delivery and perinatal outcome. European Journal of Obstetrics \& Gynecology and Reproductive Biology, 123, 188-192. http://dx.doi.org/10.1016/j.ejogrb.2005.02.026

[10] Rhoades, D.A., Latza, U. and Mueller, B.A. (1999) Risk factors and outcomes associated with nuchal cord. A population-based study. Journal of Reproductive Medicine, 44, 39-45.

[11] Ghi, T., D’Emidio, L., Morandi, R., Casadio, P., Pilu, G. and Pelusi, G. (2007) Nuchal cord entanglement and outcome of labour induction. Journal of Reproductive Medicine, 1, 57-60.

[12] Ogueh, O., Al-Tarkait, A., Vallerand, D., Rouah, F., Morin, L., Benjamin, A., et al. (2006) Obstetrical factors related to nuchal cord. Acta Obstetricia et Gynecologica Scandinavica, 85, 810-814. http://dx.doi.org/10.1080/00016340500345428

[13] Salvador, J., Cunillé, M., Lladonosa, A., Ricart, M., Cabré, A. and Borrell, C. (2001) Characteristics of pregnant women and routine antenatal care in Barcelona, 1994-1999. Gaceta Sanitaria, 15, 230-236. http://dx.doi.org/10.1016/S0213-9111(01)71552-X

[14] González-Quintero, V.H., Tolaymat, L., Muller, A.C., Izquierdo, L., O’Sullivan, M.J. and Martin, D. (2004) Outcomes of pregnancies with sonographically detected nuchal cords remote from delivery. Journal of Ultrasound in Medicine, 23, 43-47.

[15] Mastrobattista, J.M., Hollier, L.M., Yeomans, E.R., Ramin, S.M., Day, M.C., Sosa, A., et al. (2005) Effects of nuchal cord on birthweight and immediate neonatal outcomes. American Journal of Perinatology, 22, 83-85. http://dx.doi.org/10.1055/s-2005-837737

[16] Jauniaux, E., Ramsay, B., Peellaerts, C. and Scholler, Y. (1995) Perinatal features of pregnancies complicated by nuchal cord. American Journal of Perinatology, 12, 255258. http://dx.doi.org/10.1055/s-2007-994467 